Editorial

\title{
Acknowledgement to Reviewers of Veterinary Sciences in 2017
}

Veterinary Sciences Editorial Office

MDPI AG, St. Alban-Anlage 66, 4052 Basel, Switzerland

Published: 12 January 2018

Peer review is an essential part in the publication process, ensuring that Veterinary Sciences maintains high quality standards for its published papers. In 2017, a total of 65 papers were published in the journal. Thanks to the cooperation of our reviewers, the median time to first decision was 29 days and the median time to publication was 67 days. The editors would like to express their sincere gratitude to the following reviewers for their time and dedication in 2017:

\begin{tabular}{|c|c|}
\hline Akbar, Samina & Legge, Melissa \\
\hline Anderson, Kevin L. & Leroy, Baptiste \\
\hline André-Fontaine, Genevieve & Liesegang, Annette \\
\hline Armand, Martine & Linder, Deborah \\
\hline Artemiou, Elpida & Litman, Marcus \\
\hline Avery, Anne C. & Lloyd, Janice \\
\hline Avery, Paul & Luciano, Fernando \\
\hline Bacon, Heather & Lymberopoulos, Dimitrios K. \\
\hline Bai, Hua & Lyons, Leslie A. \\
\hline Bangoura, Berit & Mackenstedt, Ute \\
\hline Barua, Animesh & Madeira De Carvalho, Luis Manuel \\
\hline Baviera-Puig, Amparo & Madison-Antenucci, Susan \\
\hline Baviskar, Pradyumna & Malissiova, Eleni \\
\hline Bennett, Peter & Marrs, Carl \\
\hline Bertelloni, Fabrizio & Martini, Mina \\
\hline Bidarimath, Mallikarjun & Matthew, Susan \\
\hline Bielefeldt-Ohmann, Helle & Mayser, Peter Andreas \\
\hline Binfet, John Tyler & McBride, Anne \\
\hline Bosilevac, Mick & Meadows, Shannon \\
\hline Boswood, Adrian & Mena, Ignacio \\
\hline Boudreau, Beth & Menzies, Paula \\
\hline Bowles, Doug & Mexas, Angela Marie \\
\hline Buch, Shilpa & Milting, Hendrik \\
\hline Cadenas, Enrique & Mohamed Hafez, Hafez \\
\hline Caires, Kyle & Moore, Robert J. \\
\hline Call, Douglas R. & Morgan, Stewart \\
\hline Callanan, Sean & Mueller, Kristina \\
\hline Cambier, Carole & Mueller, Megan Kiely \\
\hline Cannon, Claire & Murphy, Brian \\
\hline
\end{tabular}


Cannon, Claire M.

Carlisle, Gretchen K.

Carlsson, Catharina

Carretón, Elena

Catry, Boudewijn

Chahory, Sabine

Chandler, Marjorie

Chen, Guiqian

Chiara, Giudice

Cho, Jeong-il

Chowdhury, Shafiqul

Cianciolo, Rachel

COLAVITA, Giampaolo

Cooke, Barbara

Cork, Susan

Dell, Colleen

Diaz, Duarte

Diesel, Alison B

Dima, Cristian

Durham, Amy C.

Duscher, Georg G.

Economou, Vangelis

Eisler, Mark

Elder, John H.

Eliades, Theodore

Emmott, Edward

Evans, Meirion

Fadok, Valerie

Fawcett, Anne

Fei, Andrew Chang-Young

Ferri, Maurizio

Flecknell, Paul

Fletcher, Jon

Fölster-Holst, Regina

Foote, Sally J.

Fouladkhah, Aliyar

Fravalo, Philippe

French, Anne

Galuppi, Roberta

Gay, Noellie

Ghosh, Anuradha

Gilding, Edward K.

Gilger, Brian C.
Murugaiyan, Jayaseelan

Narute, Purushottam

Neerukonda, Sabarinath

Nelson, Richard W

Nelson, Richard W.

Nermes, Merja

Nilsson, Ake

Noli, Chiara

Norton, Nadine

O'Handley, Ryan

Ödman, Anna

Ohara, Takahiro

Omboni, Stefano

On, Stephen

Overall, Karen L.

Owen, Helen

Packman, Wendy

Paltrinieri, Saverio

Passalaqua, Karla

Passantino, Giuseppe

Pathania, Rajneesh

Perrucci, Stefania

Pinna, Carlo

Pirrone, Federica

Plesch, Petra Nicole

Poglayen, Giovanni

Polakof, Sergio

Poli, Alessandro

Ponzio, Patrizia

Ramos, Victoria

Räsänen, Pekka Tuomas

Reddy, Sakamuri

Reinhard-Bjornvad, Charlotte

Ressel, Lorenzo

Roberta, Perego

Roosje, Petra

Sakai, Hiroki

Sakwe, Amos

Santovito, Elisa

Sanz, Ivan

Saxena, Vikas

Shen, Zhenyu

Shepherd, M. L. 
Giulivi, Cecilia

Gordon, Catherine A.

Graham, Peter

Hales, Dale Buchanan

Han, Zhe

Hassan, Sharifah Syed

Hayette, Marie-Pierre

Heinze, Cailin R.

Helmy, Yosra A.

Herrel, Anthony

Hezzell, Melanie

Hildebrandt, Nicolai

Hildreth, Blake

Hodgson, Kate

Hoenig, Margarethe

Howell, Tiffani

Humer, Elke

Jaja, Ishmael

Jang, Young Dal

Johnson, Gayle C.

Jones, Meinir G.

Jones, Philip

Kang, Ji-Houn

Keane, Orla M.

Kent, Michael S.

Ketzis, Jennifer K.

Kim, Kyung-su

Kong, Weili

Kostomitsopoulos, Nikolaos G.

Kuhne, Franziska

Kumar, Asit

Labella, Alejandro

Larsen, Jennifer

Larsen, Jennifer A.

Lashley, Victoria
Sheppard, Mary N.

Shil, Niraj

Shu, Sherry

Singh, Ravinder J.

Stefanon, Bruno

Steinbach, Sarah M. L.

Stiles, Jean

Stone, Diana

Suravajhala, Prashanth

Taboada, Eduardo

Teske, Erik

Thomas, Dave

Tidona, Flavio

Tidwell, Amy

Tonolo, Giancarlo

Tvarijonaviciute, Asta

Upadhyaya, Indu

Van Wettere, Arnaud

Verbalis, Joseph

Verstegen, Martin W. A.

Vitale, Maria

Vitztum, Coley

Vorbruggen, Gerd

Waal, Theo De

Westman, Mark

White, Amelia G.

White, Stephen David

Wiederstein-Grasser, Iris

Wynwood, Sarah J.

Yang, Vicky K.

Yang, Zhengyu

Zandvliet, M.M.J.M. (Maurice)

Zhu, Guoqiang

Zienius, Dainius

(C) 2018 by the author. Licensee MDPI, Basel, Switzerland. This article is an open access article distributed under the terms and conditions of the Creative Commons Attribution (CC BY) license (http://creativecommons.org/licenses/by/4.0/). 